\title{
MODELLING AND STUDY OF A MICROWAVE PLASMA SOURCE FOR HIGH- RATE ETCHING
}

\author{
S. Pauly ${ }^{1}$, A. Schulz 1 , M. Walker ${ }^{1}$, M. Gorath², K.-M. Baumgärtner ${ }^{2}$, G.E.M. Tovar ${ }^{1}$ \\ ${ }^{1}$ Institute of Interfacial Process Engineering and Plasma Technology (IGVP), \\ University of Stuttgart, Stuttgart, Germany \\ ${ }^{2}$ Muegge GmbH, Reichelsheim (Odenwald), Germany \\ Steffen.Pauly@igvp.uni-stuttgart.de
}

\begin{abstract}
The aim of this study is to investigate and to optimize an existing microwave-powered remote plasma source (RPS) with respect to the etching rate and gas temperature and to simplify the setup to save production costs. To achieve these goals, a FEM-based model of the RPS has been developed in order to investigate the microwave coupling into the plasma chamber and the microwave field distribution as well as the plasma itself. Different examples of FEM-based microwave simulations at different conditions and their experimental validations will be presented.
\end{abstract}

Keywords: Microwave Plasma, Remote Plasma Source, Modelling, Plasma Etching.

\section{Introduction}

Photoresists are photo-susceptible polymer layers used in the semi-conductor industry to manufacture surface structures in the sub-micrometer range. This polymer layer acts as shaping die for the envisaged microstructures and has to be removed in the final step of the manufacturing process. Here, conventional wet etching of the cured resist pattern is not a process of choice, as the microstructures must not be damaged, which is not guaranteed with wet etching processes. In contrast, dry plasma chemical etching by means of radicals generated in the plasma chamber of a remote plasma source (RPS) is a suitable method for avoiding damages to the microstructures made of metals like nickel, copper or gold. Another advantage is, that the products does not have to be dried and cleaned after the manufacturing process and can be directly processed.

Plasma is often called the fourth state of matter, because usually every matter goes through the known states of matter from solid to liquid to gaseous as the energy input is raised. By further increasing the energy input, atoms and molecules become partly ionized and molecules dissociate until the plasma state arises. Hence, plasma is an ionized gas in which the same amount of negative charge in the form of electrons exists as positive charge in the form of ions. When a high-frequency electrical field is applied, only the electrons can follow the fast electromagnetic waves. Thus, only the electrons can gain energy. It's then called a nonequilibrium, or non-isothermal plasma, which means that the electron temperature is much higher than the gas and the ion temperature $\left(T_{e} \gg T_{i} \approx T_{g}\right)$. The advantage of such a non-isothermal plasma is that high temperatures are avoided, and thus even sensitive surfaces can be etched. However, the accelerated electrons collide with neutral atoms and molecules, which leads to excited atoms, excited molecules, ions or radicals. During plasma etching, the highly reactive radicals then react with the substrate surface atoms forming gaseous molecules. The plasma etching is a purely chemical etching process. A schematic view of the etching process is shown in figure 1.

The aim of the study is to investigate and to optimize the RPS with respect to the etching rate and gas temperature. Therefore, a finite element method (FEM) based plasma model of 
the RPS has been developed to investigate the microwave coupling into the plasma chamber and the microwave field distribution as well as the plasma itself.

\section{Experimental Setup}

Figure 1 shows a schematic view of the Remote Plasma Source (RPS) flanged on top of a reactor. The RPS consists of a magnetron with a frequency of $2.45 \mathrm{GHz}$ and a power up to $3 \mathrm{~kW}$, and waveguides to feed the microwaves from the magnetron into the plasma chamber. The operating pressure in the reactor and the RPS is between $1 \mathrm{~Pa}$ and $150 \mathrm{~Pa}$. There is atmospheric pressure in the waveguides. A vacuum window made of ceramic is between the rectangular waveguide and the plasma chamber. Together with the tuning stubs and the mode coupler, the configuration is such that the plasma ignites and operates without readjusting the microwave coupling. A quartz glass window is arranged on the top of the RPS for optical investigations of the plasma. The gas used in this work is oxygen, hence the resulting products of the etching process are not dangerous for the environment such that no waste gas treatment is needed. The gas is fed in above the plasma chamber via a mass flow controller. One important advantage of the RPS is its compact design, so that it can be easily flanged on existing production lines.

To validate the numerically calculated microwave field configuration in the plasma chamber when there is no plasma in the RPS (ignition condition), polymethylmethacrylate (PMMA) plates were placed into the plasma chamber and heated up with the microwave. After removing the plates from the RPS, the heat distribution was visualized with a thermal camera system and liquid crystal sheets and compared with the numerical simulation.

To validate the electric field distribution when there is plasma in the RPS (operational condition), photographs through the observation window were taken and compared with the electric field distribution calculated with the later explained Drude model.

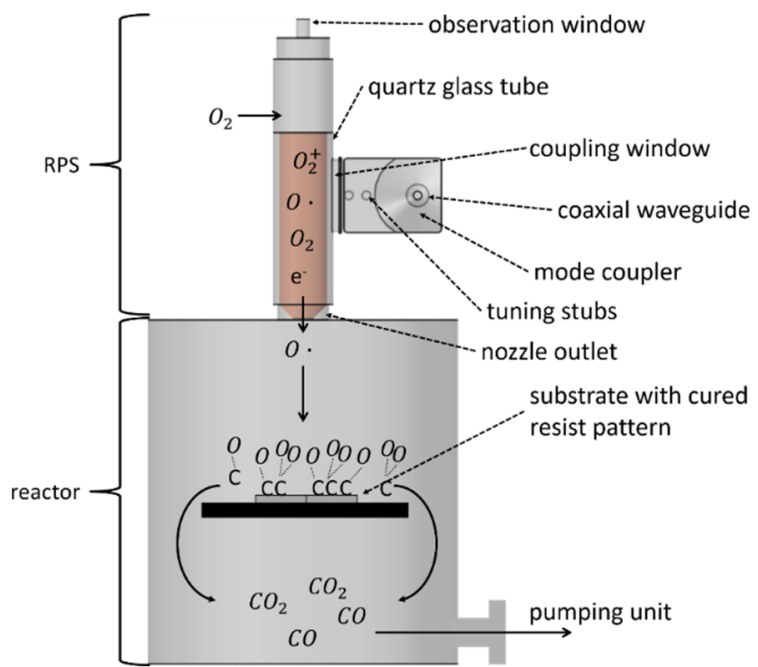

Figure 1: Schematic view of the plasma etching process of the cured resin.

The resin on top of the substrate is etched by oxygen radicals generated by microwaves in the orange-colored plasma chamber of the RPS. 


\section{Simulation Setup}

To investigate and to optimize the RPS, a 3D FEM model based on the engineering drawings of the RPS was developed which is shown in figure 2. For the investigation of this study, there are two essential process conditions of the plasma source. The first one is the ignition condition of the RPS when the microwave power is turned on, but there is no plasma in the chamber yet, so the microwaves propagate like in free space. The second one is the operational condition when the plasma has been ignited. In this case the plasma affects the microwave field distribution in the RPS. In the following section, the equations for both conditions as well as the boundary conditions for the calculations will be presented.

To calculate the electromagnetic wave propagation at the ignition condition, the wave equation for the electric field $\vec{E}$ has to be solved:

$$
\nabla \times\left(\frac{1}{\mu_{r}} \nabla \times \vec{E}\right)-k_{0}^{2} \epsilon_{r} \vec{E}=0,
$$

with the relative permeability $\mu_{r}$, the vacuum wave number $k_{0}$ and the relative permittivity $\epsilon_{r}$ which is $\epsilon_{r}=1$ for vacuum.

For the operational condition of the RPS, when a plasma is assumed, eqn. (1) is complemented with the Drude model. As mentioned in the introduction, only the electrons in the plasma can follow the high-frequent electromagnetic waves. This behavior can be treated like the free electron gas in a metal which the Drude model [1] describes. However, eqn. (1) has to be adapted with a frequency-dependent permittivity $\epsilon(\omega)$. By converting the equation of motion of an electron $e$ with the mass $m_{e}$, an expression for the conductivity $\sigma(\omega)$ containing the electron density $n_{e}$ can be derived as follows [2]:

with the collision frequency [3]

$$
\sigma(\omega)=\frac{e^{2} n_{e}}{m_{e}(v+i \omega)^{\prime}}
$$

$$
v=v_{e N}=n_{g a s} \overline{v_{e}} \sigma_{e N} .
$$

The collision frequency depends on the mean velocity of an electron $\bar{v}_{e}$, the neutral gas density $n_{g a s}$ and the collision cross section $\sigma_{e N}$. Inserting eqn. (2) into the mathematical term for the permittivity with the plasma frequency known as [4]

$$
\omega_{p}=\sqrt{\frac{n_{e} e^{2}}{m_{e} \epsilon_{0}}}
$$

the permittivity becomes [5]

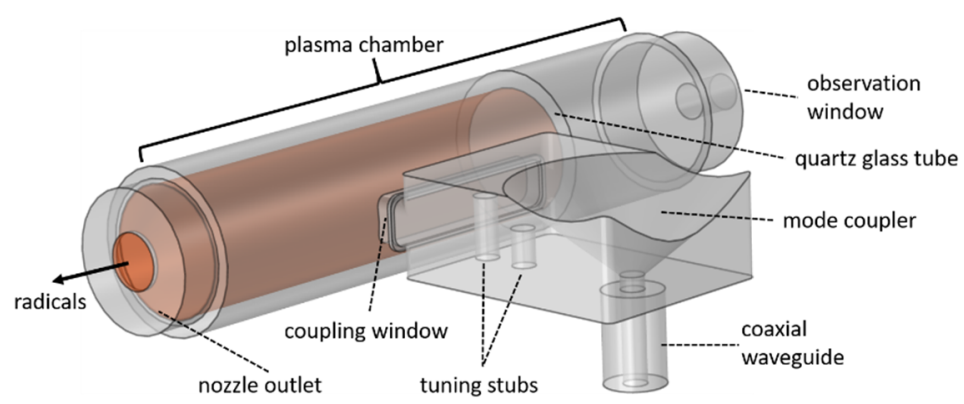

Figure 2: Schematic image of the RPS. The orange-colored volume shows where the plasma exists and, where the Drude model is implemented in the simulation. 


$$
\epsilon(\omega)=1-\frac{i \sigma(\omega)}{\omega \epsilon_{0}}=1-\frac{\omega_{p}^{2}}{\omega^{2}\left(1-\frac{i v}{\omega}\right)} .
$$

$i$ is the imaginary number and $\epsilon_{0}$ the vacuum permittivity. This leads to the frequencydependent wave equation for the electric field:

$$
\nabla \times\left(\frac{1}{\mu_{r}} \nabla \times \vec{E}\right)-k_{0}^{2}\left(\epsilon_{r}-\frac{i \sigma(\omega)}{\omega \epsilon_{0}}\right) \vec{E}=0 .
$$

If the frequency $\omega$ and the plasma frequency $\omega_{p}$ are equal, the cut-off density can be derived from eqn. (4) as follows:

$$
n_{\text {cutoff }}=\frac{m_{e} \epsilon_{0}}{e^{2}} \cdot \omega_{p}^{2}
$$

If collisions in the plasma are neglected, the cut-off density represents a threshold that indicates whether the injected microwaves are affected by the plasma or not. Is the electron density below the cut-off density, the electromagnetic waves can enter the plasma. If the electron density exceeds the cut-off density, the wave gets repelled. This effect will be presented in the results and can be seen in figure 5.

In the finite element method, the boundary conditions are the setting of known values on specific nodes, lines or areas. In the case of the RPS (figure 2), all walls enclosing the simulation domain are set as perfect electric conductors, so that there are no losses considered. The nozzle outlet is defined as scattering boundary condition, which is transparent for incoming scattered waves. The coaxial waveguide, where the microwaves are fed into the system, is set as a coaxial port with an input power of $3 \mathrm{~kW}$ and a microwave frequency of $2.45 \mathrm{GHz}$.

\section{Results and Discussion}

In the following section, the simulations at ignition conditions with the experimental validations will be presented at first and the results of the simulations under operational conditions with its validations discussed afterwards.

Ignition condition means that there is no plasma in the RPS but only the electric field. In order to do so, the whole geometry of the RPS is represented as free space according to eqn. (1). Figure 3 shows the distribution of the calculated normalized electric field

$$
E_{\text {norm }}=|\bar{E}|=\sqrt{E_{x}^{2}+E_{y}^{2}+E_{z}^{2}}
$$




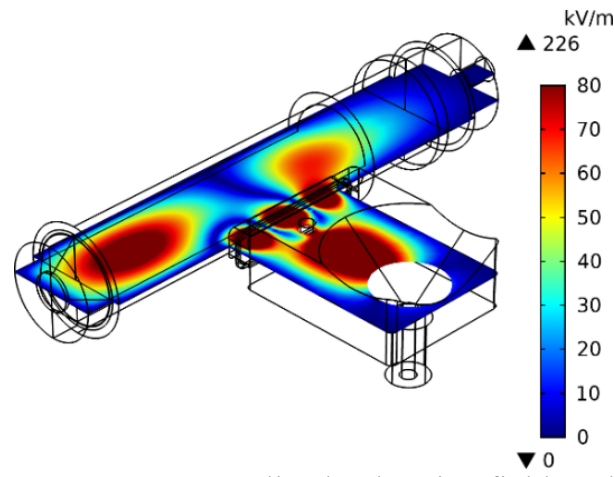

Figure 3: Normalized electric field. The electric field distribution is calculated for ignition conditions, so no plasma is inside the plasma chamber of the RPS.

In the RPS. The microwave is fed into the system via the coaxial waveguide and then tuned with the mode coupler and the tuning stubs for coupling into the plasma chamber through the vacuum window. Figure 3 shows a high electric field above the tuning stubs due to the permanent ignition and operational condition configuration. Because of the high relative permittivity of the material of the vacuum window, the propagation velocity of the microwave is reduced, leading to three field maxima. In the plasma chamber are two field maxima, a higher one near the nozzle and a smaller one at the top. To validate the calculated electric field distribution in the plasma chamber at ignition conditions, a PMMA plate was placed into the plasma chamber of the RPS in an experiment and implemented in the model which is shown in figure 4 a).

In the experiment, the PMMA plate was heated up for 30 seconds with the microwave power of $\mathrm{P}=3 \mathrm{~kW}$. After removing the plate from the RPS, the heat distribution was visualized by two different methods, a thermal camera system b) and liquid crystal sheets c). Liquid crystal sheets consist of crystals 3 to $5 \mu \mathrm{m}$ in size which are dispersed within a polymer matrix. The crystals exhibit a color spectrum response with the change of temperature [6]. Because of the different visualization systems in the experiment and the associated color spectra of them, the different colors in b) and c) can be neglected. Only the position of the maxima and their geometry are considered. As can be seen, the images of the experiment and the image of the simulation show a high maximum at the top and a smaller one at the bottom. On the right side of the images b), c), d), where the microwaves are fed into the system, all three images show a hotspot, too. Considering the time dependence of the experiment, the geometry of the electric field distribution in the experiment fits very well with the calculated field distribution at ignition conditions.

As mentioned before, there are two conditions which are essential for that study. The following simulations were performed under operational conditions, which means that there is plasma in the RPS. For simulations with plasma, the frequency-dependent conductivity $\sigma(\omega)$ has to be included into eqn. (1), which leads to eqn. (6). The frequency-dependent wave equation is determined by the electron density which can be used as a parameter to define a plasma and to implement it into the simulation model [4]. Eqn. (6) is valid in the volume of the orange-colored inner part of the plasma chamber in figure 2, whereas eqn. (1) is valid for the remaining simulation model of the RPS. To investigate the frequency dependence of the assumed plasma, the electron density is then varied around the 


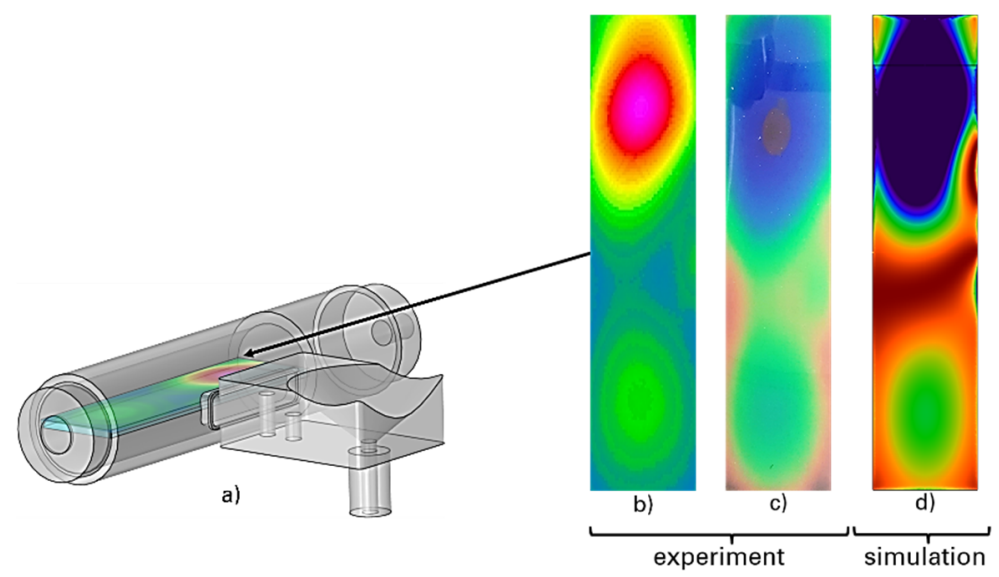

Figure 4: Integrated PMMA substrate in the RPS simulation model a). To compare the electric field distribution: PMMA substrate heated-up with $3 \mathrm{~kW}$ microwave power for 30 seconds and visualized with b) infrared camera system, c) liquid crystal sheet and in comparison, d) the calculated field.
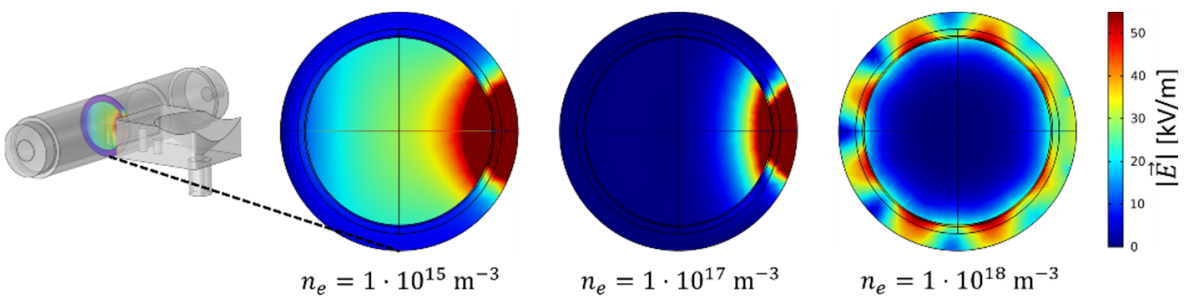

Figure 5: Normalized electric field in a cross section of the plasma chamber at operational conditions for different electron densities.

cut-off density of the plasma. In addition, collisions are considered by defining a collision frequency, see eqn. (3). The collision frequency is determined by the neutral gas density $n_{\text {gas }}$, a typical electron velocity $\overline{v_{e}}$ and the collision cross section $\sigma_{e N}$. The considered collision frequency for oxygen is $v=9.31 \cdot 10^{7} \mathrm{~s}^{-1}$, with the assumed values for operational condition: $n_{\text {gas }}=1.94 \cdot 10^{22} \mathrm{~m}^{-3}, \quad \bar{v}_{e}=1.06 \cdot 10^{6} \frac{\mathrm{m}}{\mathrm{s}}$ and $\sigma_{e N}=4.5$. $10^{-20} \mathrm{~m}^{2}$. Langmuir probe and double probe measurements will follow to validate the assumed data and to implement an electron density distribution profile in the simulation model. Figure 5 shows the normalized electrical field $E_{\text {norm }}$ of a cross section of the plasma chamber at different electron densities, where the microwaves are fed in the system from the right side. The cut-off density at $2.45 \mathrm{GHz}$ is $7.45 \cdot 10^{16} \mathrm{~m}^{-3}$. For the electron density below the cut-off density at $n_{e}=1 \cdot 10^{15} \mathrm{~m}^{-3}$, the microwaves can penetrate the plasma area comparable to figure 3 , where no plasma is assumed. If the cut-off density is exceeded, the microwaves are repelled, and the electric field gets displaced by the plasma which can be seen at $n_{e}=1 \cdot 10^{17} \mathrm{~m}^{-3}$. By further increasing the electron density to the value $n_{e}=1$. $10^{18} \mathrm{~m}^{-3}$, which can be assumed for the RPS, the microwaves get displaced out of the plasma chamber and propagate through the quartz glass tube like in a waveguide around the plasma chamber. At that density the electric field in the quartz glass tube has eight maxima. The plasma is field-free, except for a small penetration depth which is the plasma skin depth. 
To validate the simulations with the implemented Drude model, photos of the plasma were taken through the observation window on the top of the RPS. The gas which is used for the experiments is oxygen at a pressure of $100 \mathrm{~Pa}$ and a gas flow of $3 \mathrm{slm}$. The microwave has a frequency of $2.45 \mathrm{GHz}$ and $3 \mathrm{~kW}$ power. The photo of the plasma can be seen in figure 6 a). It shows eight bright shining regions arranged in a circle.

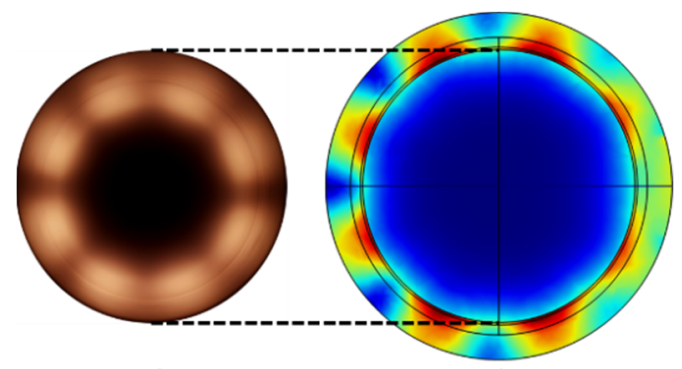

a)

b)

Figure 6: Photo of an oxygen plasma a) compared with the normalized electric field $b$ ) of the calculated RPS at operational conditions for $n_{e}=1 \cdot 10^{18} \mathrm{~m}^{-3}$.

To compare the photo of the experiment with the simulations, b) shows again the normalized electric field $E_{\text {norm }}$ simulated under operational conditions at an electron density of $n_{e}=$ $1 \cdot 10^{18} \mathrm{~m}^{-3}$. As already mentioned, at that density the microwaves propagate through the quartz glass tube around the plasma, and the field distribution has eight maxima. Figure 6 a) only shows the plasma chamber of the RPS but not the quartz glass tube. So, the section of the photo a) corresponds to the inner area of the simulation b) which is indicated by the dashed lines. It suggests that at the eight maxima of the electric field around the plasma area, the plasma shines brighter, because there is also more energy available for the ionization and excitation. To confirm that assumption, more detailed simulations will follow in that study based on experimental investigations with Langmuir probe and double probe measurements of the plasma.

\section{Conclusion}

To investigate the remote plasma source regarding its two essential operating states, the ignition condition and the operational condition, a FEM based simulation model has been developed. The ignition condition represents the simulation case, when there is no plasma in the RPS yet. For that condition, the electric field distribution was calculated with the wave equation in free space. To simulate the RPS under operational conditions, which means there is plasma existing, the wave equation was adapted with a frequency dependent conductivity based on the Drude model.

In order to validate the simulations under ignition conditions, PMMA plates were heated up in the RPS, and the heat distribution was visualized with different methods. The heat distribution was then compared with the calculated electric field distribution on a PMMA plate, which was integrated in the model. The idea behind this comparison was that at positions of higher electric field the PMMA plate should be heated up more than at positions of lower electric field. The results show that the experiment and the simulation are in good agreement. 
The simulation with the implemented Drude model shows at the assumed electron density an electric field distribution with eight maxima arranged circular around the plasma chamber. In order to validate this simulation, photos of the plasma were taken and compared with the simulations of the normalized electric field. The plasma photo shows a structure with eight bright shining regions arranged circular in the plasma chamber, which suggests that they correspond to the eight electric field maxima around the plasma chamber in the simulation. To confirm that assumption, further investigations of the plasma with Langmuir probe and double probe measurements as well as optical investigations with the infrared camera system will follow.

\section{References}

1. Drude, P.: Annalen der Physik. Zur Elektronentheorie der Metalle, 1900, 306: 566-613.

2. Janzen, G., Plasmatechnik: Grundlagen, Anwendungen, Diagnostik, 1992, 3.

3. Bergmann, L., Schaefer, C., Lehrbuch der Experimentalphysik: VielteilchenSysteme, 1992, $\mathbf{5}$.

4. Gaiser, S., Diagnostik und Modellierung eines Mikrowellen-Plasmabrenners bei Atmosphärendruck, Dissertation, University of Stuttgart, 2017.

5. Moisan, M., Pelletier, J., Plasma Technology, 4, Microwave Excited Plasmas, Elsevier, Amsterdam-London-New York-Tokyo, 1992.

6. Edmund Optics, Temperature Sensitive Liquid Crystal Sheets: https://www.edmundoptics.com/f/temperature-sensitive-liquid-crystalsheets/11878/ [Date accessed 05/06/19]. 